\title{
Poor rectal absorption of trimethoprim/sulphamethoxazole in treating Pneumocystis carinii pneumonia
}

\author{
ROBERT T. DORR \\ M.S. \\ J. ROBERT Powell \\ Pharm.D. \\ MeREDITH HeICK \\ M.D. \\ DAVID W. BARRY \\ M.D.

\begin{abstract}
From the College of Pharmacy and Department of Internal Medicine, University of Arizona, Health Sciences Center, Tucson, AZ, and Burroughs Wellcome Co., Research Triangle Park, N.C.
\end{abstract}

\begin{abstract}
Summary
A 24-year-old female with Hodgkin's disease and Pneumocystis carinii pneumonia was treated with trimethoprim/sulphamethoxazole (TMP/SMX) tablets. Because treatment failure was feared owing to chronic emesis potentially resulting in incomplete drug absorption, the same TMP/SMX dose was administered by rectal suppositories after the 5th day of oral dosing. The relative fractions (rectal/oral) of the suppository dose absorbed for TMP and SMX were $3.0 \%$ and $19.5 \%$ respectively. When TMP/SMX treatment is required and the oral route is not practical, the investigational i.v. preparation should be obtained.
\end{abstract}

\section{Introduction}

The combination of trimethoprim and sulphamethoxazole (Septra ${ }^{\circledR}$, Bactrim ${ }^{\circledR}$ ) appears to be an effective and reasonably safe treatment for immunosuppressed patients with documented or suspected Pneumocystis carinii pneumonia (Lau and Young, 1976). It is also effective as a prophylactic regimen for special patient populations at high risk of developing $P$. carinii pneumonia (Hughes et al., 1977) and has decided advantage over earlier, more toxic drugs such as pentamidine isethionate (Western, Perera and Schultz, 1970). The recommended dosage schedule is trimethoprim $20 \mathrm{mg}$ and sulphamethoxazole $100 \mathrm{mg} / \mathrm{kg}$ body weight/day administered by mouth in 4 equally divided doses for 14 days. These doses produce mean serum concentrations of approximately $4-10 \mathrm{mg} / 1$ trimethoprim and $80-120 \mathrm{mg} / 1$ sulphamethoxazole (Hughes, Feldman and Sanyal, 1975). Although there is no clear association of these drug levels to successful treatment, Lau and Young noted that the 2 histologically

Requests for reprints should be addressed to: Robert $T$. Dorr, M.S., Department of Pharmacy and Supply, University of Arizona Health Sciences Center, Tucson, Arizona 85724. confirmed failures of $8 \mathrm{TMP} / \mathrm{SMX}$-treated patients occurred when the treatment period was less than 10 days and the serum drug levels one hr after dosing were low (i.e. TMP $1.5 \mathrm{mg} / \mathrm{l}$ and SMX 20-21 mg/l) (Lau and Young, 1976). The presence of ileus may have accounted for 2 treatment failures in this series owing to incomplete drug absorption.

The following case report describes an immunosuppressed cancer patient with documented $P$. carinii pneumonia treated with the recommended TMP/SMX oral doses for 5 days. Because of persistent nausea and vomiting the authors were concerned that the drugs were not being adequately absorbed. As a result, the patient was changed to the same daily drug doses administered as extemporaneously compounded rectal suppositories. The relative bioavailability of TMP and SMX by the oral and rectal routes is reported herein.

\section{Case report}

A 24-year-old, 51-kg woman with nodular sclerosing Hodgkin's disease (stage II-E) was admitted to the Arizona Health Sciences Center for a suspected respiratory infection. Chest examination revealed symmetrical breath sounds with no rales or rhonchi. Temperature was $38.8^{\circ} \mathrm{C}$. Serum creatinine was 0.6 $\mathrm{mg} / \mathrm{dl}$ and did not change significantly for the duration of her stay in hospital. Chest X-rays showed no infiltrate. On the second hospital day a temperature spike occurred $\left(39.8^{\circ} \mathrm{C}\right)$, cultures were obtained, and antibiotics started (gentamicin $60 \mathrm{mg}$ i.v. every $8 \mathrm{hr}$, cephapirin $2 \mathrm{~g}$ i.v. every $6 \mathrm{hr}$, carbenicillin $5 \mathrm{~g}$ i.v. every $4 \mathrm{hr}$ ). Low grade fever persisted, however, and on the 4th hospital day a temperature spike of $39 \cdot 2^{\circ} \mathrm{C}$ was noted. Diffuse rales and rhonchi were now present. Chest $\mathrm{X}$-ray showed extensive consolidation in most lung fields as well as pleural capping in both lung fields. Arterial blood gases on room air were $P_{O_{2}} 39.9 \mathrm{mmHg}, P_{C_{2}} 27 \cdot 1$, and 
pH 7.5. Bronchoscopy was done and bronchial washings were positive for $P$. carinii on Giemsa methenamine silver stain. The patient was treated with 240 mg TMP and $1200 \mathrm{mg} \mathrm{SMX}$ as tablets given by mouth every $6 \mathrm{hr}$. On the 5 th hospital day the patient defervesced, and antimicrobials other than TMP/ SMX were stopped. However, persistent nausea and frequent vomiting which started the day after TMP/ SMX therapy was begun necessitated multiple repeat doses of the TMP/SMX. By the 7th hospital day there was extensive clearing of the infiltrates on $X$-ray. Arterial blood gases were now $\mathrm{PO}_{2} 76 \mathrm{mmHg}$, $\mathrm{PCO}_{2} 29, \mathrm{pH} 7 \cdot 48$. On the 9th hospital day (5th day of TMP/SMX treatment) emesis was such a problem that treatment failure was feared and rectal administration was considered. Since the rectal absorption of TMP/SMX was not known to the authors, blood samples for TMP/SMX assay were obtained just before the last oral dose and at hourly intervals over one dosage interval (6 hr) (Fig. 1). After the last blood sample the same daily TMP/SMX dose was administered as a suppository containing $800 \mathrm{mg}$ SMX and $160 \mathrm{mg}$ TMP inserted rectally every $4 \mathrm{hr}$. The suppositories were extemporaneously com-

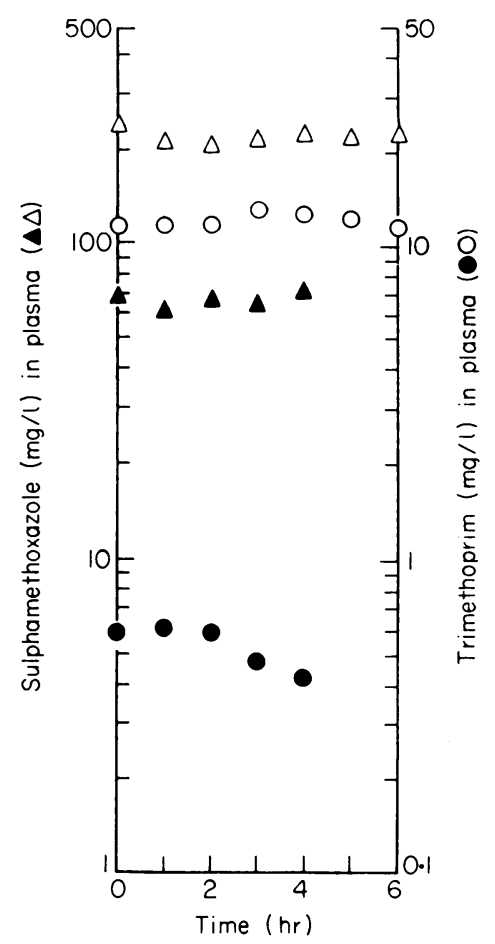

FIG. 1. Trimethoprim and sulphamethoxazole plasma concentrations over a dosing interval following administration of tablets by mouth (unfilled symbols) and rectal extemporaneously compounded suppositories (filled symbols). pounded in the hospital pharmacy using a cocoa $\frac{2}{3}$ butter base and finely ground tablets of the drug. The $\frac{\varrho}{c}$ suppositories were well tolerated and premature $\stackrel{\leftrightarrow}{\Rightarrow}$ expulsion was not noted during the remainder of the $\overrightarrow{\overrightarrow{\vec{S}}}$ stay in hospital. Blood samples for TMP/SMX assay $\overline{0}$ were obtained immediately before and 1, 2, 3, and 4 듬 $\mathrm{hr}$ after the last dose in hospital (Fig. 1). At the time $\frac{\bar{\omega}}{\bar{D}}$ of discharge on the 13th hospital day, the patient $\overparen{\mathbb{}}$ was supplied with enough suppositories to complete a 14-day course of therapy. Other than a brief upper respiratory infection thought to be of viral origin, $\vec{\circ}$ the patient has remained well for the subsequent 3 months.

Plasma samples for TMP and SMX assay were analysed by Wellcome Research Laboratories. This assay utilizes modifications of the microbiological or technique of Bushby and Hitchings (1968) for TMP. and of the Bratton and Marshall chemical method for sulphonamides (Bratton et al., 1939).

\section{Results}

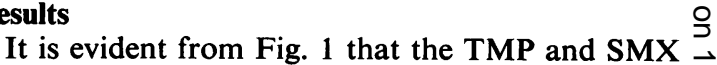
serum concentrations produced by the oral regimen $T$ were certainly adequate in spite of frequent emesis. Drug plasma levels obtained from the suppositories were extremely low. Since these drug plasma lever 2 described a dosing interval which can be assumed to $\overrightarrow{0}$ approximate steady-state conditions, the relative bioavailability of the drugs from the suppository car be calculated using the equation:

$$
\frac{\text { AUC suppository }}{\text { AUC tablet }}
$$

where $F_{\text {rel }}$ is the relative fraction of the dose absorbed using the tablet as the standard for comparison and AUC is the area under the plasma concentration-time curve at steady-stage using the trapezoidal rule (Gibaldi and Perrier, 1975). Thus the relative fractions (rectal/oral) of the suppository dose absorbed for TMP and SMX were $3.0 \%$ and $19.5 \%$ respectively.

\section{Discussion}

Fortunately, this patient appears to have been adequately treated before changing to suppositories. It is possible that the low levels of TMP/SMX were adequate to treat her infection, although this is unlikely. Initially it was feared that emesis was interfering with TMP/SMX absorption and thought was given to obtaining an investigational preparation of TMP/SMX for i.v. infusion. This, however, was rejected in favour of the rectal route because previous cancer chemotherapy had sclerosed many easily accessible peripheral veins and 2 antecubital cut-down sites created during this admission had been difficult to maintain. 
Conclusions from this report are constrained by the use of an extemporaneously prepared dosage form in a single severely ill patient. However, it is felt that the extremely poor rectal absorption of the drug relative to equivalent oral dosing in a typically life-threatening illness justifies a report at this time. Based on this information and their inability to find other published data, the authors recommend that rectal administration of TMP/SMX be avoided. This case also demonstrates that nausea and vomiting are not necessarily associated with inadequate drug absorption. In centres where $\boldsymbol{P}$. carinii pneumonia is not rare a rapid laboratory assay of TMP and SMX would be helpful. When trimethoprim and sulphamethoxazole are required and the oral route is not available or desirable, it is recommended that the investigational i.v. preparation be obtained. This parenteral preparation has been successfully and safely used in the treatment of other infections in cancer patients (Grose, Bodey and Loo, 1979).

Although there are many clinical situations which result in the need for an extemporaneously compounded dosage form, the clinician must recognize that there may be a risk of altered bioavailability influencing clinical response.

\section{References}

Bratton, A.C., Marshall, E.K., Babbitt, D. \& HendRICKSON, A.R. (1939) A new coupling component for sulfanilamide determination. Journal of Biological Chemistry, 128, 537.

BushbY, S.R.M. \& Hitchings, G.H. (1968) Trimethoprim, a sulphonamide potentiator. British Journal of Pharmacology and Chemotherapy, 33, 72.

Gibaldi, M. \& Perrier, D. (1975) Pharmacokinetics, p. 293. Marcel Dekker, New York, etc.

Grose, W.E., Bodey, G.P. \& Loo, T.L. (1979) Clinical pharmacology of intravenously administered trimethoprim/sulfamethoxazole. Antimicrobial Agents and Chemotherapy, 15 (3), 447.

Hughes, W.T., Feldman, S. \& Sanyal, S.K. (1975) Treatment of Pneumocystis carinii pneumonitis with trimethoprim-sulfamethoxazole. Canadian Medical Association Journal, 112, 475.

Hughes, W.T., Kuhn, S., Chaudhury, S., Feldman, S., Verzosa, M., Aur, R.J., Pratt, C. \& George, S.L. (1977) Successful chemoprophylaxis for Pneumocystis carinii pneumonitis. New England Journal of Medicine, 297, 1419.

LAU, W.K. \& YounG, L.S. (1976) Trimethoprim-sulfamethoxazole treatment of Pneumocystis carinii pneumonia in adults. New England Journal of Medicine, 295, 716.

Western, K.A., Perera, D.R. \& Schultz, M.G. (1970) Pentamidine isethionate in the treatment of Pneumocystis carinii pneumonia. Annals of Internal Medicine, 73, 695. 